\title{
X Congreso Ibérico de Agroingeniería

\section{Análisis y optimización del diseño inicial de las barras de un pulverizador hidráulico suspendido de 26 metros mediante el Método de los Elementos Finitos}

\author{
H. Malón' ${ }^{1}$, N. Angosto ${ }^{1}$, M. Vidal1 ${ }^{1}$, F.J. García-Ramos ${ }^{1}$, J. Cuartero' ${ }^{1}$, A. Bone ${ }^{1}$ \\ 1 Universidad de Zaragoza; hml@unizar.es; 681633@unizar.es; vidalcor@unizar.es; fjavier@unizar.es; \\ jcs@unizar.es; anbone@unizar.es
}

\begin{abstract}
Resumen: Los pulverizadores hidráulicos de barras son equipos muy extendidos que se emplean para la aplicación de productos fitosanitarios (herbicidas, insecticidas y fungicidas) en cultivos agrícolas. El diseño de las barras de los pulverizadores hidráulicos es uno de los aspectos más críticos en el análisis del comportamiento estructural de estos equipos, concretamente el análisis de la influencia de los efectos dinámicos generados por las irregularidades del terreno. En este artículo se analiza mediante técnicas numéricas basadas en el Método de los Elementos Finitos el comportamiento estructural de las barras de un pulverizador hidráulico suspendido equipado con barras de 26 metros de anchura de trabajo, sometidas a esfuerzos dinámicos generados por irregularidades del terreno. Para la realización del estudio han sido considerados tres casos de carga. El primero corresponde al peso propio y los dos restantes son casos de carga dinámicos que corresponden a dos irregularidades del terreno. Los resultados obtenidos de los cálculos numéricos han dado lugar a un proceso de optimización del diseño inicial, el cual se basa en criterios de rigidez y resistencia. Como conclusión del trabajo se ha obtenido el diseño de las barras de un pulverizador hidráulico suspendido con una anchura de cobertura de 26 metros, que son capaces de soportar efectos dinámicos de trabajo en campo, así como una metodología de diseño y optimización basada en el Método de los Elementos Finitos para barras de pulverizador hidroneumático de gran anchura de cobertura de pulverización.
\end{abstract}

Palabras clave: Análisis numérico; Plaguicida; Efectos dinámicos; MEF;

\section{Introducción}

Los pulverizadores hidráulicos de barras son equipos muy extendidos que se emplean para la aplicación de productos fitosanitarios (herbicidas, insecticidas y fungicidas) en cultivos agrícolas.

Según su disposición con respecto al tractor al que se van a acoplar existen tres tipos: a) los pulverizadores autopropulsados, los cuales son independientes y no requieren de ningún tractor para moverlo y realizar trabajo en campo; b) los pulverizadores arrastrados, en los cuales el depósito y las barras están colocados sobre un chasis con ruedas que está enganchado al tractor y c) los pulverizadores suspendidos, en los cuales el depósito y las barras están colgados del tractor.

En el diseño de pulverizadores de barras, uno de los aspectos más críticos es el análisis del comportamiento estructural de las barras, concretamente el análisis de la influencia de los 


\section{CONGRESO IBÉRICO DE AGROINGENIERÍA \\ X CONGRESSO IBÉRICO DE AGROENGENHARIA \\ 3 - 6 septiembre 2019, Huesca - España}

efectos dinámicos generados por las irregularidades del terreno. El problema en el diseño de las barras de estos equipos es que son estructuras en voladizo con longitudes de vuelo elevadas. Esta gran longitud de voladizo implica que los efectos inerciales en las barras debidos a movimientos verticales del tractor generados por las irregularidades en el terreno pueden dar lugar a zonas de concentración de tensiones que pueden ocasionar un fallo prematuro de la estructura resistente de la barra.

Por esta razón un correcto diseño de las barras de pulverizadores es imprescindible para evitar su fallo estructural, al mismo tiempo que permita realizar el correcto tratamiento en campo con las barras extendidas.

\section{Objetivo}

El objetivo principal del proyecto consiste en la definición de una metodología de análisis y optimización del diseño de barras de pulverizador hidráulico, basada en el Método de los Elementos Finitos (MEF). Esta metodología debe permitir analizar el comportamiento estructural de las barras sometidas a esfuerzos dinámicos generados por las irregularidades del terreno, con el fin de optimizar su diseño, evitando el fallo estructural, al mismo tiempo que se permite realizar el correcto tratamiento en campo con las barras extendidas.

Con el fin de alcanzar el objetivo principal del proyecto se ha aplicado la metodología desarrollada en el análisis y optimización del diseño inicial de las barras de 26 metros de un pulverizador hidráulico suspendido, en cuyo diseño no han sido considerados los efectos dinámicos de las solicitaciones existentes en el trabajo diario en campo.

\section{Materiales y métodos}

El trabajo realizado en el proceso de análisis y optimización del diseño inicial de las barras de un pulverizador hidráulico suspendido de 26 metros se ha basado en el análisis numérico mediante el Método de los Elementos Finitos (MEF). Esta metodología es una técnica empleada frecuentemente en el análisis estructural de máquinas y vehículos [1-9], así como en maquinaria y aperos agrícolas [10-12] A continuación se describen las fases de la metodología aplicada durante el estudio realizado.

\subsection{Modelo numérico}

El modelo a analizar en el estudio corresponde a las barras de un pulverizador hidráulico de $26 \mathrm{~m}$. En el análisis numérico se ha desarrollado un modelo que corresponde a la mitad del equipo de pulverización, debido a la simetría con respecto al eje longitudinal del tractor que presenta el pulverizador con las barras extendidas. La aplicación de condiciones de contorno de simetría en el modelo numérico permite reducir el coste computacional del análisis numérico, obteniendo resultados equivalentes al análisis con el modelo completo. El modelo numérico discretizado consta de 27.016 nodos y 25.979 elementos.

El diseño de las barras del pulverizador, el cual se muestra en la figura 1, está formado por 6 secciones, las cuales corresponden al soporte de las barras y 5 tramos de barras articuladas. En la discretización de estas secciones han sido empleados elementos tipo SHELL de 3 y 4 nodos en los perfiles estructurales, mostrados en gris en la figura 1. También han sido empleados elementos tipo BEAM, en rojo en la figura 1, en el modelado de las barras de refuerzo en cada una de las secciones de las barras, así como en la discretización de los cilindros hidráulicos y bulones. Las uniones articuladas entre la diferentes secciones que componen el modelo han sido realizadas mediante Multi Point Constrain (MPC's) con el fin evitar que las uniones sean completamente rígidas, y así poder reproducir su comportamiento real. En la figura 2 se muestra un detalle del modelo discretizado, en él se pueden observar los diferentes tipos de elementos empleados en el modelo numérico. 
X CONGRESO IBÉRICO DE AGROINGENIERÍA

X CONGRESSO IBÉRICO DE AGROENGENHARIA

3- 6 septiembre 2019, Huesca - España

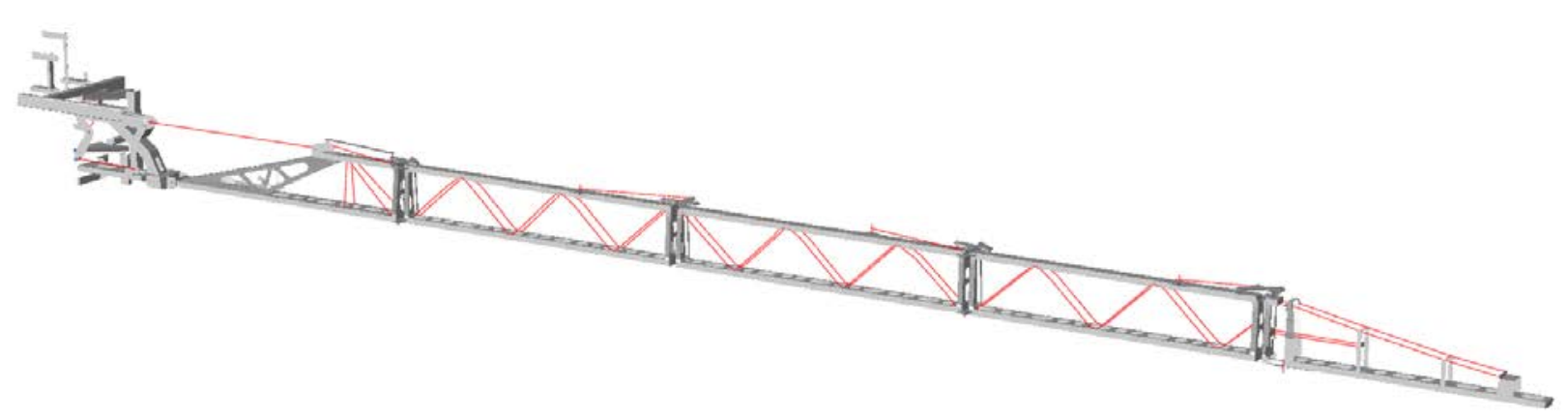

Figura 1. Modelo numérico de las barras del pulverizador hidráulico.

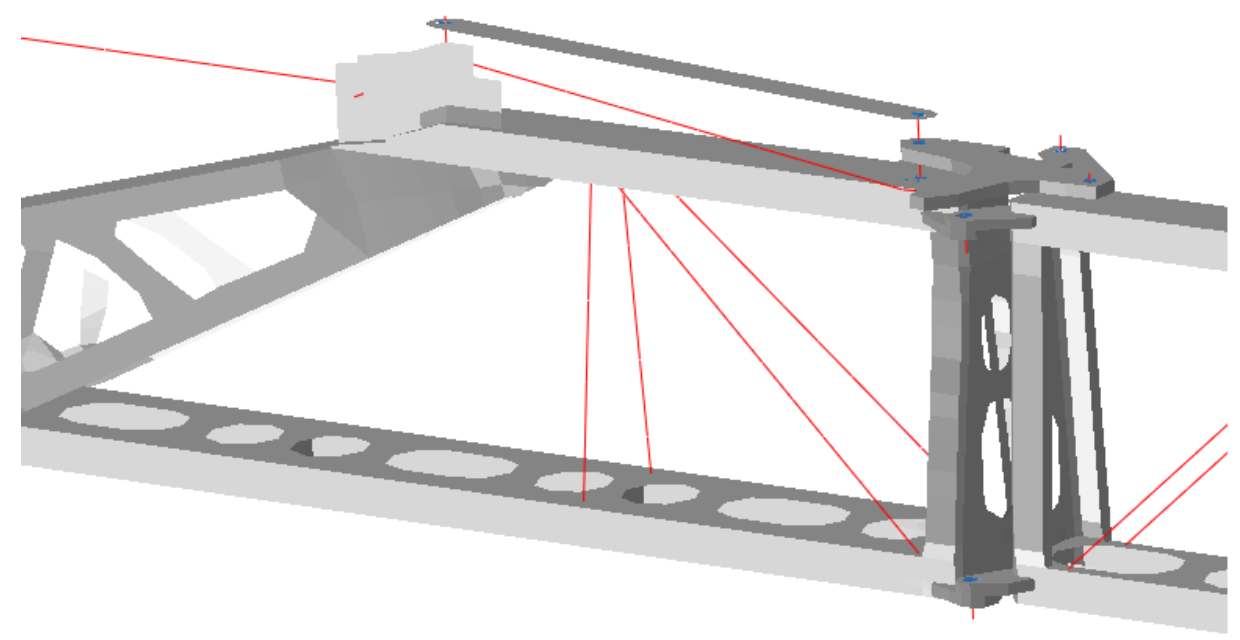

Figura 2. Detalle del modelo numérico de las barras del pulverizador hidráulico.

\subsection{Materiales}

En el análisis del diseño inicial se ha considerado que la totalidad de los componentes resistentes de la estructura son de acero S275. A los elementos no estructurales, como son los tornillos, bulones y cilindros hidráulicos, se les han asignado propiedades mecánicas de acero elástico lineal. Las propiedades mecánicas de los aceros empleados en el análisis del diseño inicial se muestran en la tabla 1.

Tabla 1. Propiedades mecánicas del acero empleado en el análisis del diseño inicial

\begin{tabular}{ccccccc}
\hline Material & $\begin{array}{c}\text { Modulo de } \\
\text { Young } \\
\mathbf{( M P a )}\end{array}$ & $\begin{array}{c}\text { Coeficiente } \\
\text { de poison }\end{array}$ & $\begin{array}{c}\text { Densidad } \\
\mathbf{( k g / \mathbf { m } ^ { 3 } )}\end{array}$ & $\begin{array}{c}\text { Límite } \\
\text { elástico } \\
\mathbf{( M P a )}\end{array}$ & $\begin{array}{c}\text { Límite } \\
\text { tracción } \\
\mathbf{( M P a )}\end{array}$ & $\begin{array}{c}\text { Elongación } \\
\mathbf{( \% )}\end{array}$ \\
\hline S275 & 210.000 & 0,3 & 7.800 & 275 & 410 & 19 \\
Acero & 210.000 & 0,3 & 7.800 & & & \\
\hline
\end{tabular}




\section{CONGRESO IBÉRICO DE AGROINGENIERÍA \\ X CONGRESSO IBÉRICO DE AGROENGENHARIA \\ 3 - 6 septiembre 2019, Huesca - España}

\subsection{Casos de carga}

En el proceso de análisis y optimización realizado han sido definidos y analizados tres casos carga, los cuales corresponden a un caso de carga estático, denominado Gravedad, y dos casos de carga dinámicos, denominados Escalón $50 \mathrm{~mm}$ y $20 \mathrm{~mm}$, los cuales reproducen los desplazamientos en el soporte de las barras del pulverizador generados por las irregularidades del terreno. A continuación, se describen en detalle los casos de carga definidos para el análisis de barras de pulverizador hidráulico de gran anchura de cobertura de pulverización.

\subsubsection{Caso de carga 1 - Gravedad}

El primer caso de carga analizado es un caso de carga estático en el cual se analiza el efecto de la gravedad en la totalidad de los componentes que constituyen la barra del pulverizador hidráulico. Por ello en este caso de carga únicamente se aplica la gravedad.

\subsubsection{Caso de carga 2 - Escalón $50 \mathrm{~mm}$}

El segundo caso de carga reproduce el desplazamiento generado en la barra del pulverizador por una irregularidad del terreno, concretamente se aplica un escalón de $50 \mathrm{~mm}$ de altura en el soporte de la barra del pulverizador, el cual se asciende en 0,1 segundos. Para la reproducción de esta maniobra en los cálculos numéricos ha sido definido un caso de carga formado por tres steps dinámicos. En el primero de ellos se ha aplicado únicamente la gravedad durante 1 segundo. En el segundo step se ha introducido el escalón de $50 \mathrm{~mm}$, mediante un desplazamiento vertical ascendente de $50 \mathrm{~mm}$ en 0,1 segundos en los nodos del plano de simetría, seguido de un desplazamiento de igual magnitud y sentido opuesto, con la misma duración. Para finalizar se define un tercer step de 2 segundos de duración, en el cual actúa únicamente la fuerza de la gravedad, con el fin de poder analizar el comportamiento dinámico de la estructura de barras ante el escalón generado en el segundo step.

\subsubsection{Caso de carga 3 - Escalón $20 \mathrm{~mm}$}

El tercer caso de carga definido corresponde a un escalón de $20 \mathrm{~mm}$ aplicado en el soporte de la barra del pulverizador generado por una irregularidad del terreno. La definición del caso de carga es similar al caso de carga de Escalón de $50 \mathrm{~mm}$, con la diferencia que los desplazamientos introducidos en el segundo step son de $\pm 20 \mathrm{~mm}$ en lugar de los $\pm 50 \mathrm{~mm}$ del caso de carga 2 .

\subsection{Condiones de contorno}

Las condiciones de contorno impuestas en el análisis numérico corresponden principalmente a condiciones de simetría con respecto al centro del pulverizador hidráulico, nodos mostrados en azul en la figura 3. Concretamente han sido restringidos en los nodos de simetría los desplazamientos respecto al eje longitudinal de la barra, así como los giros en torno del eje vertical y del eje transversal de la misma. Adicionalmente, para mejorar la convergencia de los cálculos numéricos, han sido restringidos los desplazamientos en la dirección del eje transversal de la barra en los nodos de simetría.

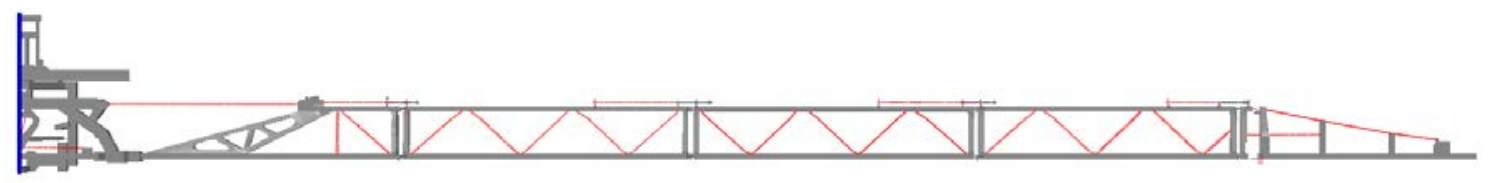

Figura 3. Nodos en los que aplican las condiciones de contorno y los casos de carga. 


\section{CONGRESO IBÉRICO DE AGROINGENIERÍA \\ X CONGRESSO IBÉRICO DE AGROENGENHARIA \\ 3 - 6 septiembre 2019, Huesca - España}

Para finalizar, en el caso de carga de gravedad han sido restringidos los desplazamientos verticales en los nodos de la superficie de simetría.

\section{Resultados modelo inicial}

Una vez han sido definidos la geometría, los materiales, los casos de carga y las condiciones de contorno, se han realizado los cálculos numéricos del modelo inicial mediante técnicas basadas en el MEF.

A continuación, se muestran los resultados de desplazamientos y tensiones de Von Mises obtenidos de los tres casos de carga analizados del diseño inicial de las barras de un pulverizador hidráulico.

\subsection{Caso de carga 1 - Gravedad}

En referencia a los desplazamientos verticales obtenidos en el caso de carga de Gravedad, los cuales se muestran en la figura 4 , se observa que el desplazamiento máximo se localiza en el extremo de la barra del pulverizador, obteniendo se un desplazamiento máximo de $143 \mathrm{~mm}$.

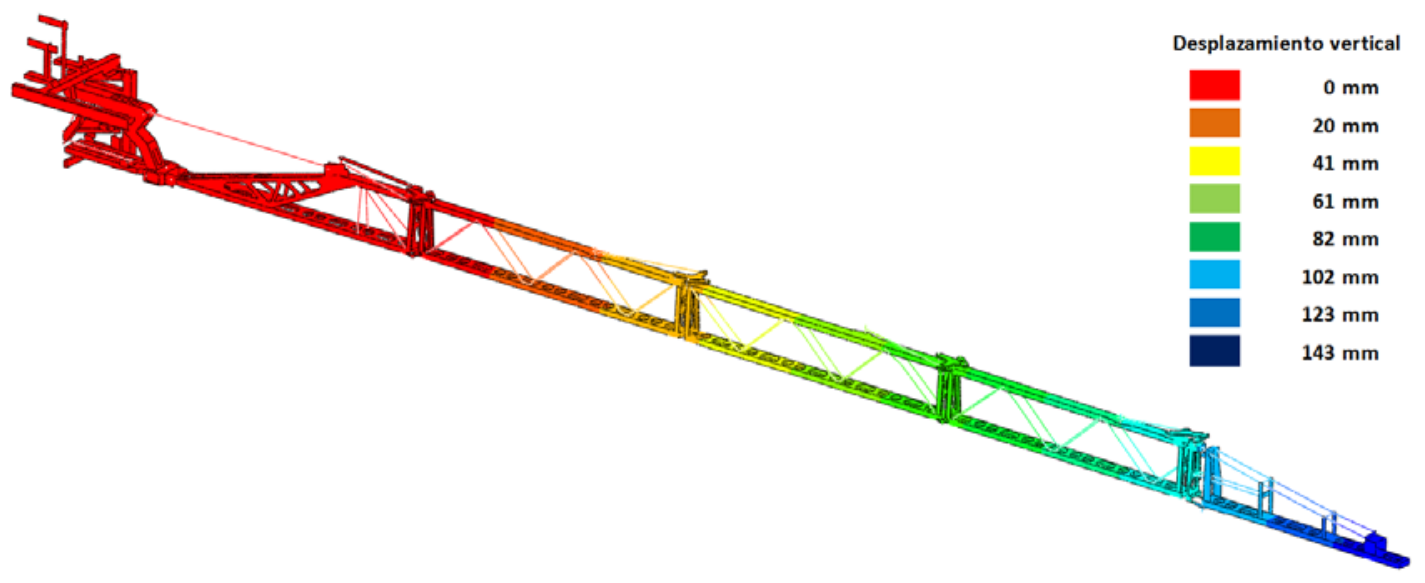

Figura 4. Desplazamiento vertical (mm) en el caso de carga Gravedad.

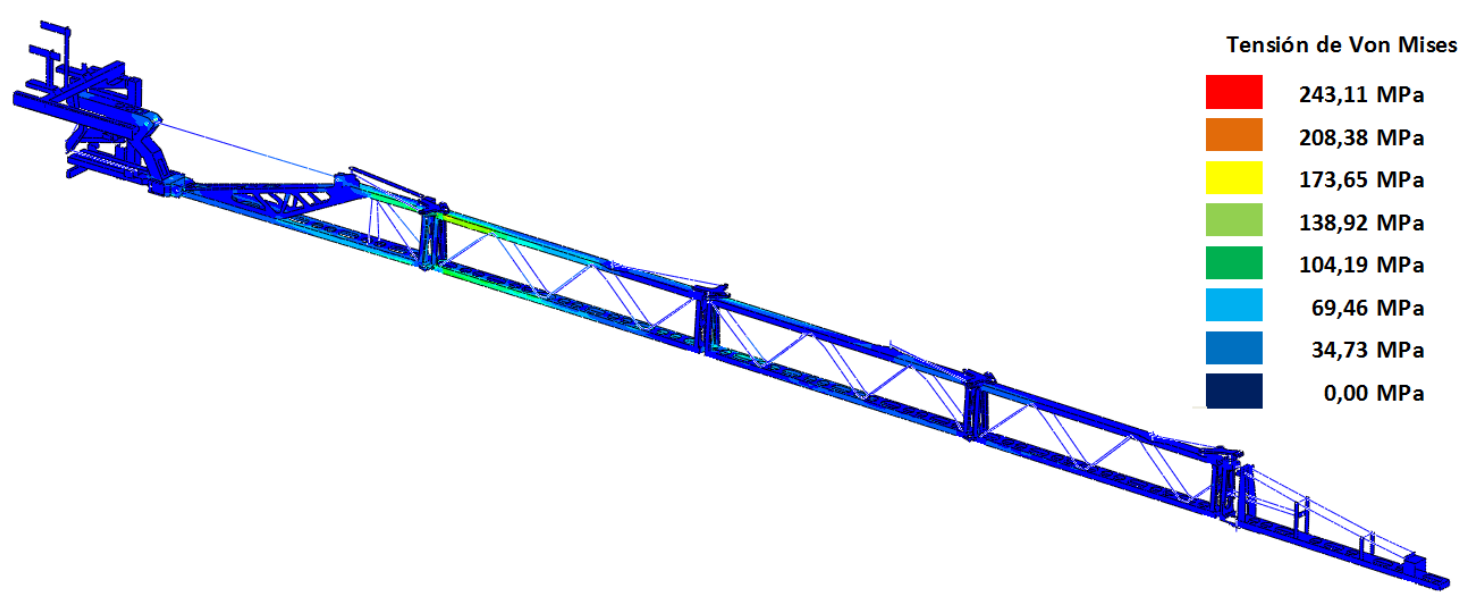

Figura 5. Tensiones de Von Mises. (MPa) en el caso de carga Gravedad.

Por otro lado, las tensiones de Von Mises más altas se localizan en las secciones 1 y 2 , concretamente en las zonas cercanas a la unión entre ambos componentes, como se muestra en 


\section{CONGRESO IBÉRICO DE AGROINGENIERÍA \\ X CONGRESSO IBÉRICO DE AGROENGENHARIA \\ 3 - 6 septiembre 2019, Huesca - España}

la figura 5. La localización de las tensiones máximas es debida a que la sección 1 es un componente más rígido, debido a su geometría y la unión mediante el cilindro hidráulico al soporte, que la sección 2 (primera de las tres secciones centrales de la barra). Por esta razón la flexión en la barra del pulverizador comienza en esta unión, como se evidencia en la figura 4 de desplazamientos verticales. La tensión de Von Mises máxima obtenida alcanzan un valor de 243,11MPa en el perfil superior de la sección 2 de las barras, como se muestra en la figura 5, obteniéndose un coeficiente de seguridad con respecto al límite elástico del material de 1,13.

\subsection{Caso de carga 2 - Escalon $50 \mathrm{~mm}$}

El segundo caso de carga analizado corresponde a un escalón de $50 \mathrm{~mm}$ aplicado en el soporte de las barras del pulverizador, generado por una irregularidad del terreno. Este caso de carga ha sido definido como una sucesión de tres steps dinámicos con una duración total de 4,1 segundos. En la figura 6 se muestra la evolución temporal de los desplazamientos verticales de nodos pertenecientes a cada una de las secciones del modelo numérico. En la evolución temporal de desplazamientos se observa como el primer step, en el cual únicamente se aplica la gravedad, tiene una duración de 1 segundo. A continuación, se introduce el escalón mediante dos desplazamientos consecutivos de igual magnitud y sentido opuesto, con una duración total de 0,2 segundos. Desde el segundo 1,2 hasta el final del cálculo se muestra evolución del comportamiento de la barra sometida únicamente la fuerza de la gravedad.

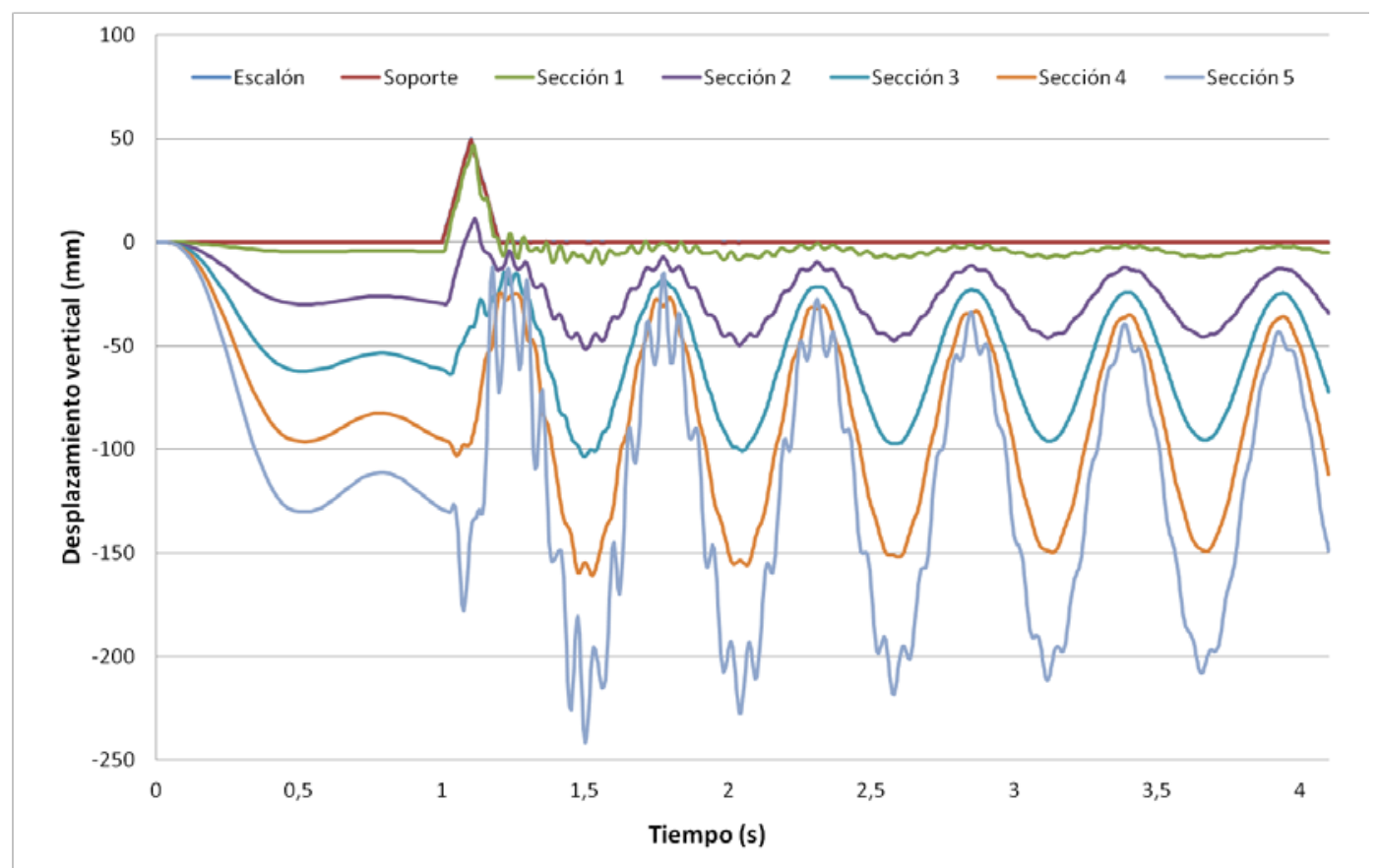

Figura 6. Evolución temporal de los desplazamientos verticales $(\mathrm{mm})$ en el caso de carga Escalón $50 \mathrm{~mm}$.

Analizando la evolución temporal de los desplazamientos verticales se observa que el desplazamiento máximo en la fase de aplicación de la gravedad se produce a los 0,5 segundos de comenzar el cálculo, volviendo al punto de desplazamiento máximo transcurrido 1 segundo de cálculo. En esta fase los desplazamientos verticales máximos se localizan en el extremo de la barra alcanzado una flecha máxima de $130 \mathrm{~mm}$.

En referencia al comportamiento de la estructura después del escalón, se observa como los desplazamientos máximos se localizan en el extremo de la barra, llegando hasta los $241 \mathrm{~mm}$, reduciéndose la magnitud de los desplazamientos en las diferentes secciones conforme están 


\section{CONGRESO IBÉRICO DE AGROINGENIERÍA \\ X CONGRESSO IBÉRICO DE AGROENGENHARIA \\ 3 - 6 septiembre 2019, Huesca - España}

más cerca del soporte. Adicionalmente, analizando la diferencia del comportamiento de las diferentes secciones que componen la estructura de las barras se aprecia una diferencia de comportamiento entre la sección 1, que apenas presenta variaciones de desplazamiento vertical, y el resto de secciones de la barra, en la cuales estos desplazamientos presentan mayor magnitud. Estos resultados permiten verificar que la flexión en la barra comienza a ser significativa a partir de la sección 2 de la estructura.

Analizando las tensiones de Von Mises se obtiene que el perfil superior de la sección 2 es el componente donde se registran los valores máximos, como se muestra en la figura 7 , alcanzando 275,14 $\mathrm{MPa}$, lo que supone un coeficiente de seguridad con respecto al límite elástico del material de 1,00 .

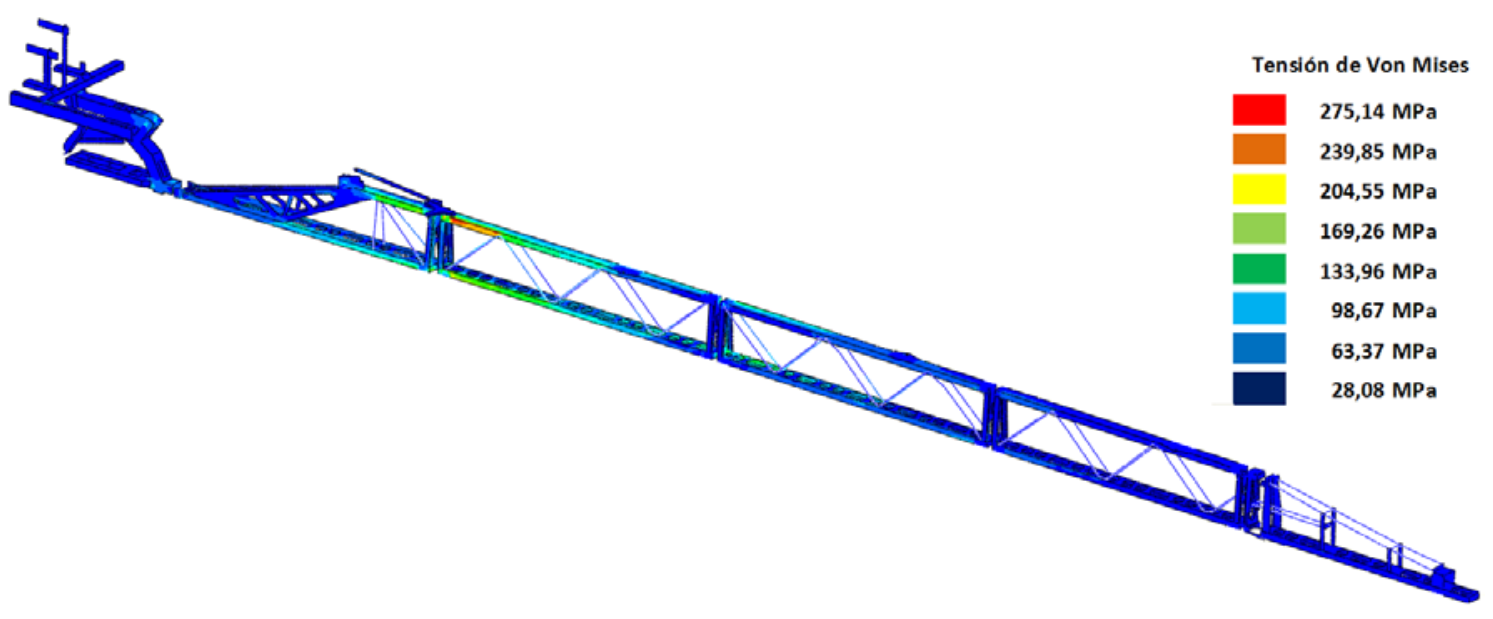

Figura 7. Tensiones de Von Mises. (MPa) en el caso de carga Escalón 50mm.

\subsection{Caso de carga 3 - Escalon $20 \mathrm{~mm}$}

El análisis del modelo inicial concluye con el caso de carga de Escalón de 20mm. Este caso de carga es análogo al caso de carga 2 con la excepción de la magnitud del escalón introducido, la cual en este caso es de $20 \mathrm{~mm}$ en lugar de $50 \mathrm{~mm}$. Por otro lado, en función de los resultados obtenidos del caso de carga de Escalón de $50 \mathrm{~mm}$, la duración del primer step, en el cual se aplica únicamente la gravedad, ha sido reducida de 1 segundo a 0,5 segundos, obteniéndose un desplazamiento vertical máximo al final de esta fase de $130 \mathrm{~mm}$, como se muestra en la figura 8 . Esta reducción de tiempo implica un gran ahorro del conste computacional en los cálculos numéricos, al mismo tiempo que se mantiene el valor máximo de desplazamiento vertical en el primer step del cálculo numérico.

Los resultados obtenidos evidencian un comportamiento similar de la barra del pulverizador al caso de carga de Escalón 50mm, variando los valores numéricos de desplazamientos y tensiones de Von Mises. Concretamente los desplazamientos máximos debidos al efecto del escalón se localizan en el extremo de la barra, obteniéndose un valor máximo de $174 \mathrm{~mm}$. Por otro lado las tensiones máximas de Von Mises se obtienen en el perfil superior de la sección 2, siendo los valores máximos de $254,05 \mathrm{MPa}$, lo que supone un coeficiente de seguridad con respecto al límite elástico del material de 1,08. 


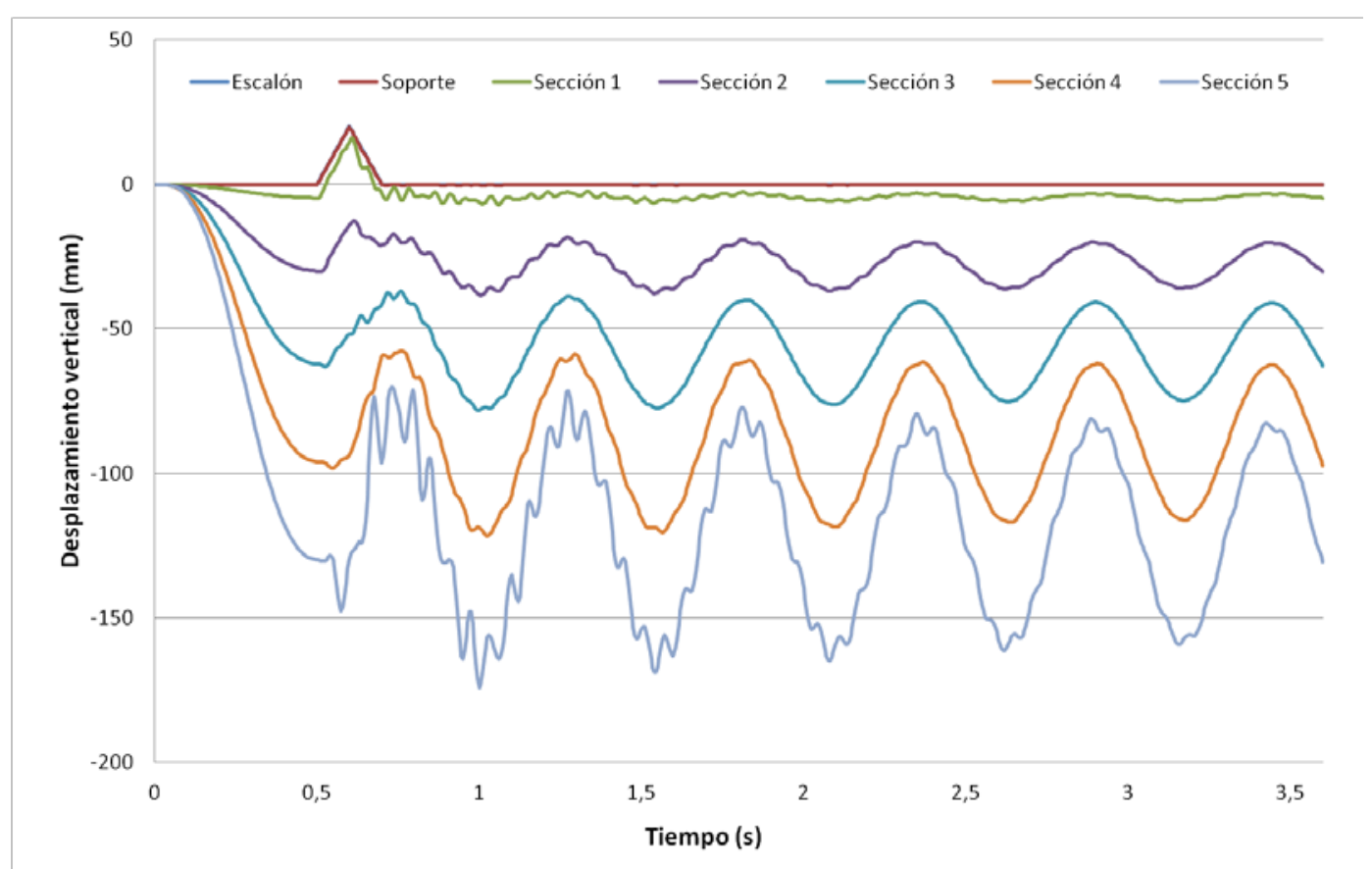

Figura 8. Evolución temporal de los desplazamientos verticales ( $\mathrm{mm}$ ) en el caso de carga Escalón 20mm.

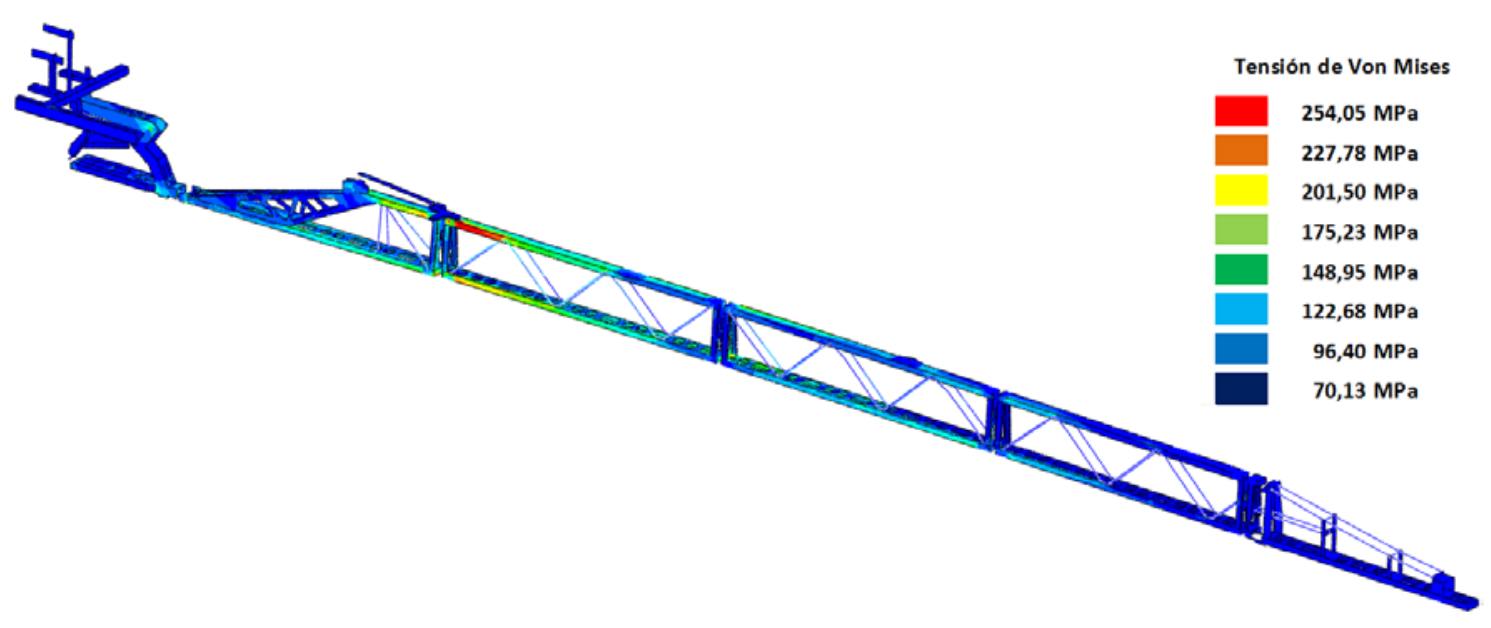

Figura 9. Tensiones de Von Mises. (MPa) en el caso de carga Escalón 20mm.

\section{Optimización del modelo inicial}

Una vez ha finalizado el análisis del diseño inicial de las barras de un pulverizador hidráulico de 26 metros, y en función de los resultados obtenidos, se ha realizado un proceso de optimización del diseño inicial con el fin de conseguir un coeficiente de seguridad de 3 en la totalidad de los componentes de la estructura para el caso de carga 3, Escalón de 20mm.

El caso de carga 3 ha sido seleccionado para el proceso de optimización por presentar unos valores de aceleración de $4 \mathrm{~g}$ frente a los $10 \mathrm{~g}$ del caso de carga 2, valores que se obtienen partir de la distancia del escalón y el tiempo empleado en recorrerlo, siéndolo los valores de aceleración vertical registrados en ensayo en campo cercanos a $2 \mathrm{~g}$ [13]. La aceleración de $4 \mathrm{~g}$ es superior al obtenido de forma experimental, lo que permite desarrollar un diseño ausente de fallo. Por otro lado la definición del criterio de coeficiente de seguridad mínimo de 3 con 


\section{CONGRESO IBÉRICO DE AGROINGENIERÍA \\ X CONGRESSO IBÉRICO DE AGROENGENHARIA \\ 3 - 6 septiembre 2019, Huesca - España}

respecto al límite elástico, corresponde a un criterio de diseño con el que se pretende evitar el fallo por fatiga de la estructura debido a efectos dinámicos.

En el proceso de optimización se ha desarrollado un trabajo iterativo en el cual se ha ido modificando el material y espesor de los componentes en función del coeficiente de seguridad obtenido. Concretamente el cambio de material se realizado asignando acero S275 a los componentes con coeficiente de seguridad mayor que 3, acero S355 a los componentes con coeficiente de seguridad entre 2 y 3, y acero STRENX700 a los materiales con coeficiente de seguridad menor que 2. Las propiedades mecánicas de los aceros empleados en el proceso de optimización de las barras del pulverizador hidráulico se muestran en la tabla 2. En los casos en los cuales el coeficiente de seguridad presentaba un valor numérico próximo a 2 ó 3 , en vez de cambiar el material del componente se ha incrementado el espesor del mismo.

Tabla 2. Propiedades mecánicas del acero empleado en el proceso de optimización

\begin{tabular}{ccccccc}
\hline Material & $\begin{array}{c}\text { Modulo de } \\
\text { Young } \\
\mathbf{( M P a )}\end{array}$ & $\begin{array}{c}\text { Coeficiente } \\
\text { de poison }\end{array}$ & $\begin{array}{c}\text { Densidad } \\
\mathbf{( k g / \mathbf { m } ^ { 3 } )}\end{array}$ & $\begin{array}{c}\text { Límite } \\
\text { elástico } \\
\mathbf{( M P a )}\end{array}$ & $\begin{array}{c}\text { Límite } \\
\text { tracción } \\
\mathbf{( M P a})\end{array}$ & $\begin{array}{c}\text { Elongación } \\
\mathbf{( \% )}\end{array}$ \\
\hline S275 & 210.000 & 0,3 & 7.800 & 275 & 410 & 19 \\
Acero & 210.000 & 0,3 & 7.800 & & & \\
S355 & 210.000 & 0,3 & 7.800 & 355 & 500 & 17 \\
STRENX700 & 210.000 & 0,3 & 7.800 & 700 & 750 & 12 \\
\hline
\end{tabular}

Analizando los desplazamientos verticales obtenidos en el modelo optimizado, los cuales se muestran en la figura 10, se observa un comportamiento similar al modelo original, pero con una reducción de $20 \mathrm{~mm}$ en la flecha máxima de la estructura y una mayor amortiguación de las oscilaciones del conjunto, lo que implica una mejora según un criterio de rigidez.

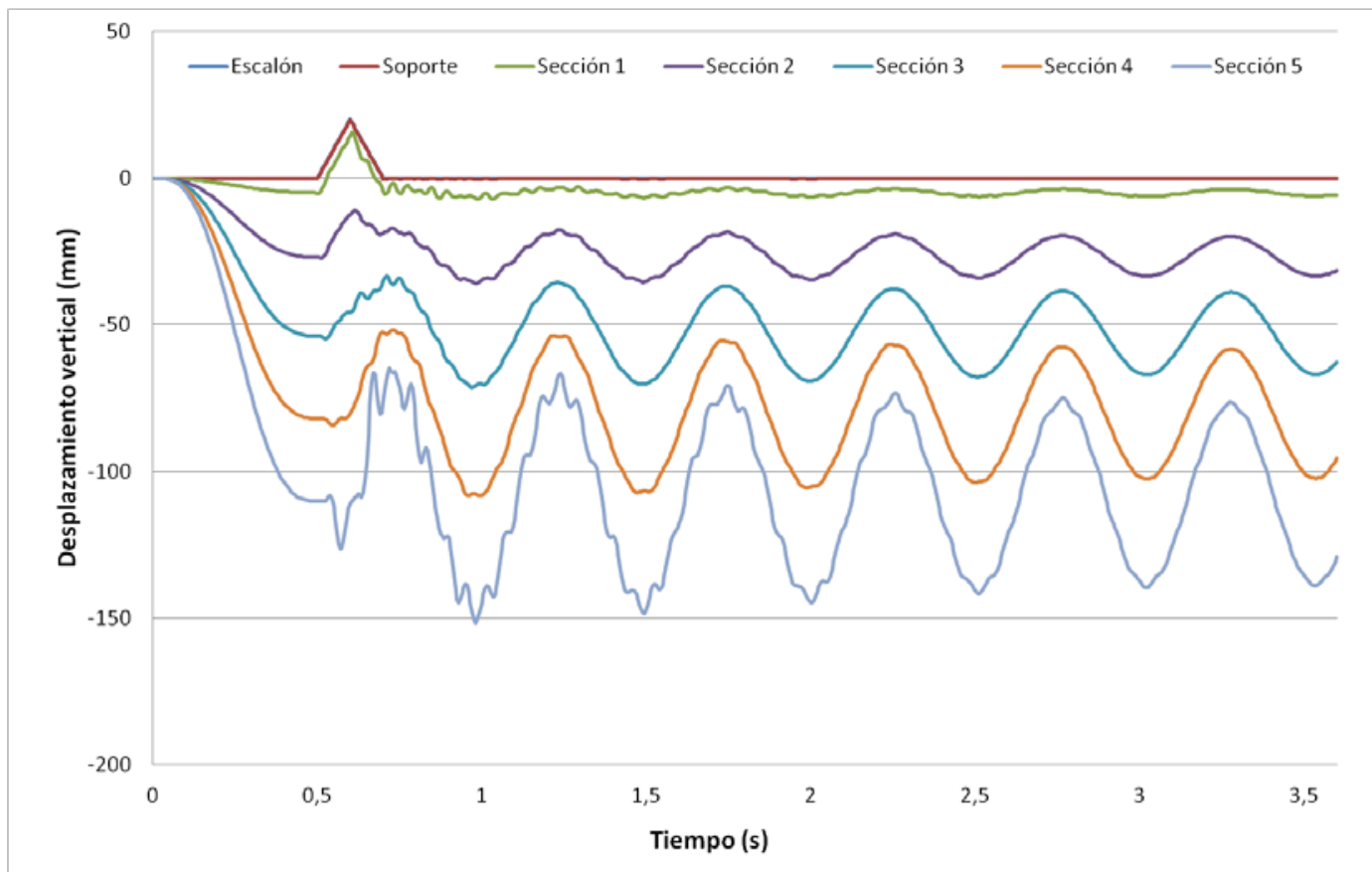

Figura 10. Evolución temporal de los desplazamientos verticales $(\mathrm{mm})$ en el caso de carga Escalón 20mm del modelo optimizado de barras de pulverizador hidráulico. 


\section{CONGRESO IBÉRICO DE AGROINGENIERÍA \\ X CONGRESSO IBÉRICO DE AGROENGENHARIA \\ 3 - 6 septiembre 2019, Huesca - España}

Desde el punto de vista de la resistencia, se ha conseguido un diseño en el cual la totalidad de los componentes que conforman la estructura de las barras tienen un coeficiente de seguridad mayor que tres, lo que permite obtener un diseño valido ante el fallo por fatiga. Los valores de tensiones de Von Mises obtenidos de los cálculos numéricos se muestran en la figura 11.

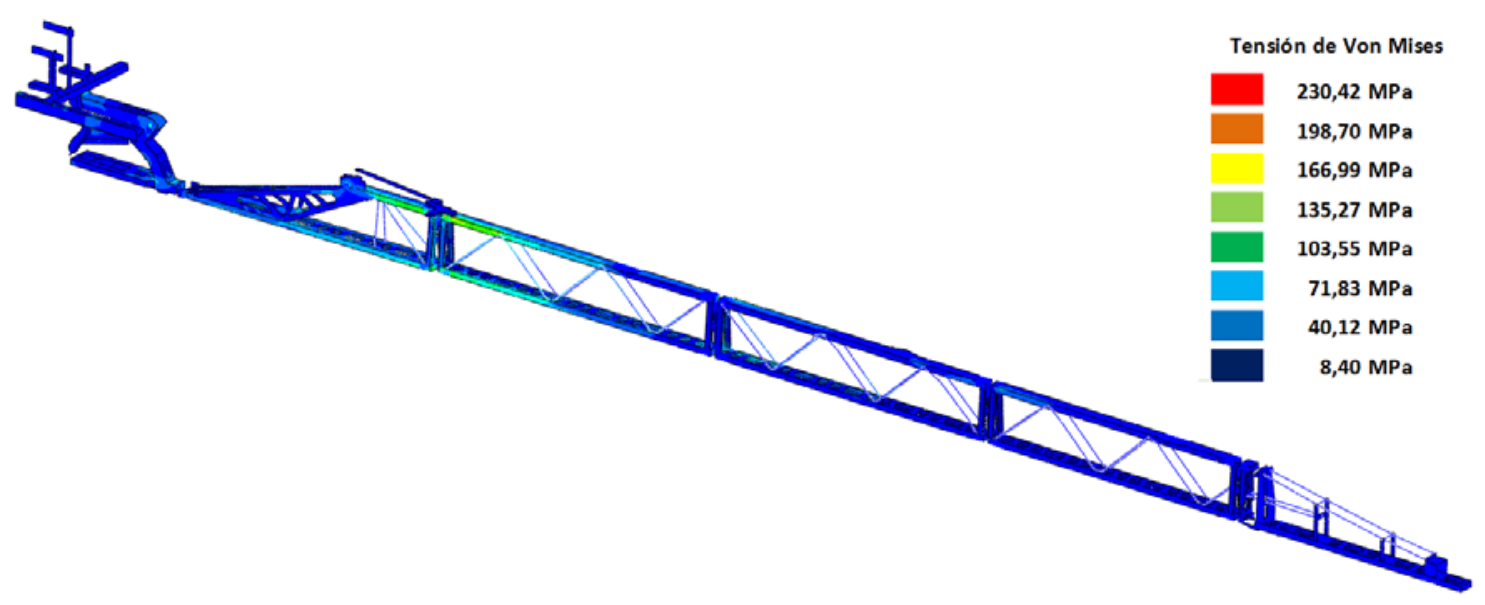

Figura 11. Tensiones de Von Mises. (MPa) en el caso de carga Escalón 20mm del modelo optimizado de barras de pulverizador hidráulico.

\section{Conclusiones}

En el estudio se ha presentado el trabajo de análisis y optimización de las barras de un pulverizador hidráulico suspendido de 26 metros de cobertura de trabajo ante solicitaciones generadas por los efectos dinámicos del trabajo en campo.

El proceso de análisis y optimización ha sido realizado mediante técnicas numéricas basadas en el Método de los Elementos Finitos (MEF), para lo que ha sido necesario la definición de la geometría, materiales, condiciones de contorno, así como casos de carga. Concretamente han sido definidos tres casos de carga, que corresponden a un caso estático de aplicación de la gravedad y dos casos dinámicos que reproducen irregularidades del terreno.

En el proceso de optimización ha sido definido el criterio seguido en la optimización del diseño inicial de las barras del pulverizador hidráulico, el cual consiste en que todos los componentes de la estructura deben presentar un coeficiente de seguridad con respecto al límite elástico del material mayor que 3 en el caso de carga de escalón de $20 \mathrm{~mm}$, con el fin de asegurar la ausencia del fallo por fatiga.

Al finalizar el proceso de optimización se ha conseguido alcanzar el objetivo del coeficiente de seguridad, mediante la aplicación de aceros S275, S355 y STRENX700, al mismo tiempo que se mejoraba el comportamiento frente a rigidez de la estructura de barras.

Para finalizar, el trabajo realizado ha permitido definir una metodología de análisis y optimización del diseño de barras de pulverizador hidráulico, basada en el Método de los Elementos Finitos (MEF), la cual permite analizar el comportamiento estructural de las barras sometidas a esfuerzos dinámicos generados por las irregularidades del terreno y optimizar su diseño. Al aplicar esta metodología se podrá evitar el fallo estructural de las barras de un pulverizador hidráulico de gran anchura de cobertura de pulverización, al mismo tiempo que se permite realizar el correcto tratamiento en campo con las barras extendidas. 


\section{CONGRESO IBÉRICO DE AGROINGENIERÍA \\ X CONGRESSO IBÉRICO DE AGROENGENHARIA \\ 3 - 6 septiembre 2019, Huesca - España}

\section{Referencias}

1. Beermann, H.J. (1984) Static Analysis of Commercial Vehicle Frames -A Hybrid Finite-Elements and Analytical Method. International Journal of Vehicle Design. 1984, 5(1-2) pp 26-52.

2. Cappello, F. Ingrassia, T. Mancuso, A. et al. Methodical Redesign of a Semitrailer. 9th International Conference on Computer Aided Optimum Design in Engineering. Computer Aided Optimum Design in Engineering IX Book Series: Wit Transactions on the Built Environment. 2005, Volume 80, pp. 359-369

3. Carrera, M. Castejon, L. Miralbes, R. et al. (2010) Behaviour IA Rear Underrun Protection System on Car-to-Tank Vehicle Impact Used for Fuel Transportation, International Journal of Heavy Vehicles Systems. 2010, 17(3-4). pp. 199-215.

4. Deng, Y.D. Wang, J. Wen, Y. et al. The Static and Dynamic Characteristics Study of Aluminum Tank Semitrailer. 2nd International Conference on Manufacturing Science and Engineering. Manufacturing Process Technology. 2011 PTS 1-5 Book Series: Advanced Materials Research, Volume 189-193, pp. 2233-2237.

5. Kodiyalam, S. \& Sobieszczanski-Sobieski, J.. Multidisciplinary Design Optimization - Some Formal Methods, Framework Requirements, and Application to Vehicle Design. International Journal of Vehicles Design. 2001, 25(1-2), pp. 3-22.

6. Karaoglu, C. \& Kuralay, N.S.. Stress Analysis of a Truck Chassis with Riveted Joints. Finite Elements in Analysis and Design. 2002, 38(12), pp1115-1130.

7. Miralbes, R. \& Castejon, L.. Fatigue Design of Tanker Semi-Trailers, Dyna. 2010, 85(6). pp. 480-488.

8. Hoefinghoff, J. Jungk, A. Knop, W. et al.. Using 3D Field Simulation for Evaluating UHF RFID Systems on Forklift Trucks. IEEE Transactions on Antennas and Propagation. 2011, 59(2), pp.689-691.

9. Miralbes, R. Malon, H \& Castejon L. (2011) Diseño de accesorios para el acoplamiento en carretillas manipuladoras: plumines y portapalets. En: Libro de resúmenes del XV International Congress on Project Engineering.2011, ISBN 978-84-615-4542-1

10. Vidal, M. Bone, A. Garcia-Ramos, F.J. Malon, H \& Villacampa, R. Desarrollo de máquina para la aplicación localizada de cebo rodenticidas en parcelas agrícolas. En: Libro de resúmenes del XV International Congress on Project Engineering.2011, ISBN 978-84-615-4542-1

11. Malon, H. Garcia-Ramos, F.J. Vidal, M. \& Bone, A. Design and optimization of a chassis for an Airassisted Sprayer with two fans using the Finite Element Method. Proyect Management and engineering research. 2014, DOI: 10.1007/978-3-319-26459-2_8

12. Sicilia, V.J. Análisis estructural de un remontador de cereales para almacenamiento de maíz. Trabajo fin de Grado. Grado en Ingeniería Agroalimentaria y del Medio Rural. Universidad de Zaragoza, 2015.

13. Jeon, Hong Young, "Instrumented Self-Propelled Sprayer to Determine Dynamic Boom Effects on Droplet Application Uniformity. " Master's Thesis, University of Tennessee, 2003. 